\title{
Yielding Behavior and Its Effect on Uniform Elongation of Fine Grained IF Steel
}

\author{
Si Gao ${ }^{1, *}$, Meichuan Chen ${ }^{1}$, Shuai Chen ${ }^{1}$, Naoya Kamikawa ${ }^{2}$, \\ Akinobu Shibata ${ }^{1}$ and Nobuhiro Tsuji ${ }^{1,3}$ \\ ${ }^{1}$ Department of Materials Science and Engineering, Kyoto University, Kyoto 606-8501, Japan \\ ${ }^{2}$ Institute for Materials Research, Tohoku University, Sendai 980-8577, Japan \\ ${ }^{3}$ Elements Strategy Initiative for Structural Materials (ESISM), Kyoto University, Kyoto 606-8501, Japan
}

\begin{abstract}
Interstitial free (IF) steel specimens with different mean grain sizes ranging from 0.4 to $12 \mu \mathrm{m}$ were fabricated by the accumulative roll bonding (ARB) process and subsequent annealing. Tensile tests at room temperature have revealed that by decreasing the mean grain size down to an ultra-fine range, the yielding behavior gradually changes from the continuous yielding to the discontinuous yielding, accompanying a yield drop phenomenon. It has been found that the yield stress of specimens having fine grain sizes shows extra-hardening, deviated from the original Hall-Petch relation for coarse-grained specimens in accordance with the discontinuous yielding. The Hall-Petch analysis also has indicated that the loss in the uniform elongation in the ultrafine grain size range is related to the appearance of the discontinuous yielding behavior. [doi:10.2320/matertrans.MA201317]
\end{abstract}

(Received August 30, 2013; Accepted October 25, 2013; Published December 6, 2013)

Keywords: yielding behavior, yield-drop, Hall-Petch relationship, uniform elongation, plastic instability

\section{Introduction}

It is well-known that the yield stress of polycrystalline metallic materials increases with decreasing the mean grain size according to the Hall-Petch relation: ${ }^{1,2)}$

$$
\sigma_{\mathrm{y}}=\sigma_{0}+k_{\mathrm{y}} d^{-1 / 2}
$$

where $\sigma_{\mathrm{y}}$ is the yield stress, $\sigma_{0}$ the friction stress, $k_{\mathrm{y}}$ a constant (Hall-Petch slope), and $d$ the mean grain size. The HallPetch relation has been established first in low carbon steels which exhibit yield point phenomenon during tensile tests. It is well-known that the yield point phenomenon in low carbon steels is caused by the dislocation locking mechanism by interstitial atoms such as carbon and nitrogen. ${ }^{3)}$ On the other hand, interstitial free (IF) steels, where carbon and nitrogen are fixed as $\mathrm{Ti} / \mathrm{Nb}$ carbides or nitrides to result in a substantially interstitial-free state, exhibit continuous yielding in the tensile test. ${ }^{4)}$ However, a recent study has revealed that discontinuous yielding, accompanying a yield drop phenomenon, occurs even in IF steels when the grain size is decreased down to an ultra-fine grain size range smaller than $2-3 \mu \mathrm{m} .{ }^{5}$ ) The present study aims to investigate the effect of yielding behavior on the Hall-Petch relation and on the ductility of fine grained IF steel.

\section{Experimental Procedure}

Ti-added commercial IF steel with the initial mean grain size of around $20 \mu \mathrm{m}$ was used in the present study. The chemical composition of the material is shown in Table 1. Starting sheets in $1 \mathrm{~mm}$ thickness, $30 \mathrm{~mm}$ width, and $300 \mathrm{~mm}$ length were subjected to 7 cycles of accumulative rollbonding (ARB) process at $500^{\circ} \mathrm{C}$ without lubrication, using $50 \%$ rolling reduction per cycle. The total strain corresponding to the ARB process was 5.6. Then, the ARB processed

*Corresponding author, E-mail: si.gao@tsujilab.mtl.kyoto-u.ac.jp
Table 1 Chemical composition of the IF steel used in this study (mass\%).

\begin{tabular}{ccccccccc}
\hline $\mathrm{C}$ & $\mathrm{N}$ & $\mathrm{Si}$ & $\mathrm{Mn}$ & $\mathrm{P}$ & $\mathrm{Cu}$ & $\mathrm{Ni}$ & $\mathrm{Ti}$ & $\mathrm{Fe}$ \\
\hline 0.002 & 0.003 & 0.01 & 0.17 & 0.012 & 0.01 & 0.02 & 0.072 & Bal. \\
\hline
\end{tabular}

sheets were annealed at different temperatures ranging from 500 to $800^{\circ} \mathrm{C}$ for $1.8 \mathrm{ks}$ in order to change the mean grain size. The microstructure of the ARB and annealed specimens was observed on longitudinal sections perpendicular to the transverse direction (TD) of the sheets by SEM-EBSD (electron back-scattering diffraction in scanning electron microscope) system and the mean grain size of the specimens was measured by the linear interception method. Tensile test specimens with $10 \mathrm{~mm}$ in gauge length and $5 \mathrm{~mm}$ in gauge width, which had a $1 / 5$ miniaturized size of the JIS-5 specimen, were cut from the ARB processed and annealed sheets. Tensile tests at ambient temperature with an initial strain rate of $8.3 \times 10^{-4} \mathrm{~s}^{-1}$ were carried out to characterize the mechanical properties of the specimens with different mean grain sizes. An extensometer was attached on the specimen during the tensile test for a precise measurement of the displacement.

\section{Results}

Specimens with mean grain sizes ranging from 0.41 to $12 \mu \mathrm{m}$ were obtained by combining ARB and subsequent annealing processes. Figure 1 shows the EBSD maps of an as-ARB specimen and ARB processed and subsequently annealed specimens. The crystallographic orientation parallel to the normal direction (ND) of the sheets is represented by the colors according to the key stereographic triangle in the figure. Low angle grain boundaries $\left(2^{\circ} \leq \theta<15^{\circ}\right)$ and high angle grain boundaries $\left(\theta \geq 15^{\circ}\right)$ are indicated by white and black lines, respectively. The as-ARB processed specimen 

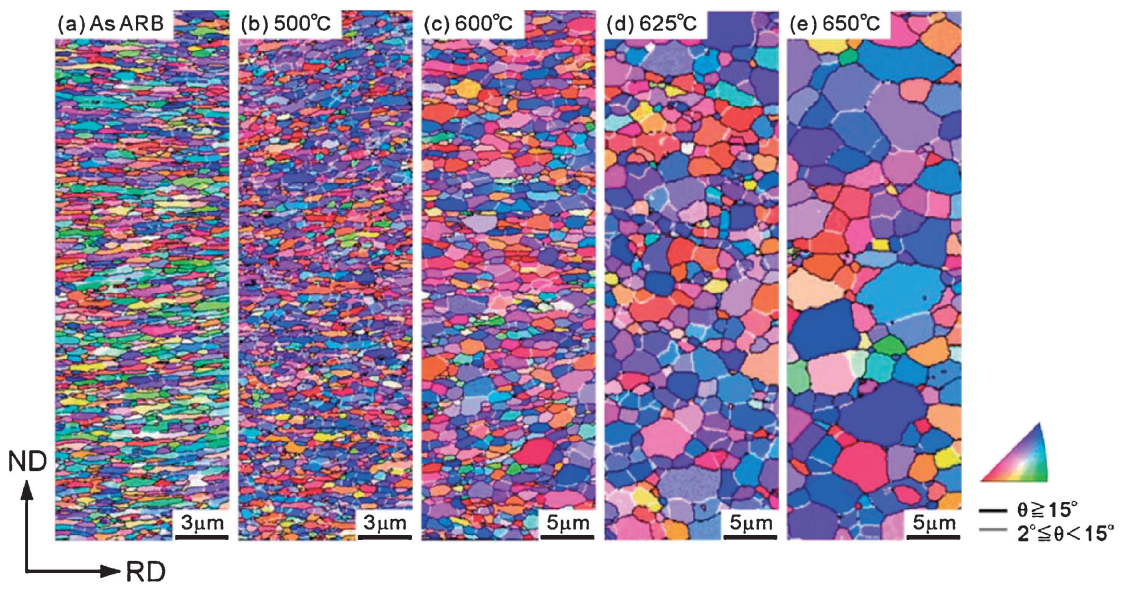

Fig. 1 EBSD maps of the IF steel ARB processed and subsequently annealed at various temperatures for $1.8 \mathrm{ks}$.

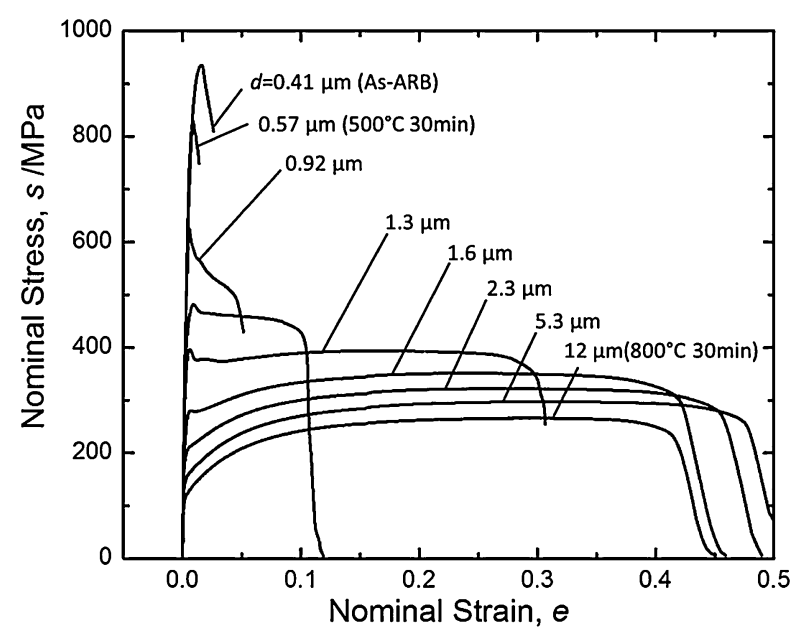

Fig. 2 Nominal stress-strain curves of the IF steel with various mean grain sizes ranging from 0.4 to $12 \mu \mathrm{m}$.

shows elongated ultrafine grains surrounded mostly by high angle grain boundaries with a mean grain size of $0.41 \mu \mathrm{m}$ in Fig. 1(a). Slight coarsening of the microstructure occurred in the specimen annealed at $500^{\circ} \mathrm{C}$ after 7 cycles $\mathrm{ARB}$, as shown in Fig. 1(b). As the annealing temperature increases, the grains gradually grew to attain more equiaxed microstructures, as shown in Figs. 1(c), 1(d) and 1(e). A weak coldrolling texture composed of $\mathrm{ND} / /\langle 111\rangle$ orientations (colored in blue) and $\mathrm{RD} / /\langle 110\rangle$ orientations (colored in red to purple) was found in these specimens. ${ }^{6,7)}$ The mean grain size, which was measured by the linear interception method in random directions, of the five specimens shown in Fig. 1, was 0.41, $0.57,0.92,1.6$ and $2.3 \mu \mathrm{m}$, respectively. For larger mean grain sizes associated with higher annealing temperatures, equiaxed microstructures were more evident.

Nominal stress-strain curves for specimens with various grain sizes are shown in Fig. 2. The mean grain size is indicated in each figure. The as-ARB processed specimen with the mean grain size of $0.41 \mu \mathrm{m}$ shows very high yield strength and a quite limited elongation. With increasing the annealing temperature (i.e., with increasing the mean grain size), the strength of the material decreased. It is very interesting that the specimens in a relatively coarse grain size range exhibit continuous yielding while the distinct yield point phenomenon characterized by a yield drop and subsequent Lüders deformation occurs for specimens in a smaller grain size range from 1.3 to $2 \mu \mathrm{m}$. The Lüders strain increases with decreasing the mean grain size from 1.6 to $1.3 \mu \mathrm{m}$. When the mean grain size was smaller than $1.3 \mu \mathrm{m}$, macroscopic necking occurred immediately after the yield point and the material led to failure. Shear band (localized deformation) was observed on those tensile specimens.

The mechanical properties obtained from tensile tests are plotted as a function of the inverse square root of the mean grain size in Fig. 3(a). The $0.2 \%$ offset proof stress $\left(\sigma_{0.2 \%}\right)$ was taken as the yield strength for the specimens exhibiting continuous yielding, while the upper yield stress was taken for those exhibiting yield point phenomenon. The Hall-Petch relation has been obtained in the coarse grain size range ( $d$ larger than $2 \mu \mathrm{m}$ ). It has been noted that the relation is associated with a significant low $k_{\mathrm{y}}\left(211 \mathrm{MPa} \cdot \mu \mathrm{m}^{1 / 2}\right)$, compared with the value reported in low carbon steels $\left(600 \mathrm{MPa} \cdot \mu \mathrm{m}^{1 / 2}\right) .{ }^{8)}$ It has been also found that the yield stress deviates from the Hall-Petch relation when the mean grain size decreases below $2 \mu \mathrm{m}$. The deviation of the yield stress became more significant as the mean grain size decreased. It is interesting that the deviated yield stress seems also to follow a Hall-Petch relation with a significantly high slope, as is indicated in the figure.

The uniform elongation of specimens is plotted in Fig. 3(b). The uniform elongation slightly increased with decreasing the grain size first and then decreased by about $5 \%$ as the mean grain size decreased from 12 to $2 \mu \mathrm{m}$. However, it sharply dropped to less than $2 \%$ within a very narrow grain size range indicated by the gray area (from about 2 to $1 \mu \mathrm{m}$ ), then leveled off when the grain size decreased below $1 \mu \mathrm{m}$.

\section{Discussion}

The yield point phenomenon in low carbon steels is usually explained by the dislocation locking mechanism by Cottrell atmosphere formed by interstitial atoms. However, the dislocation locking mechanism cannot explain the yield 
(a)
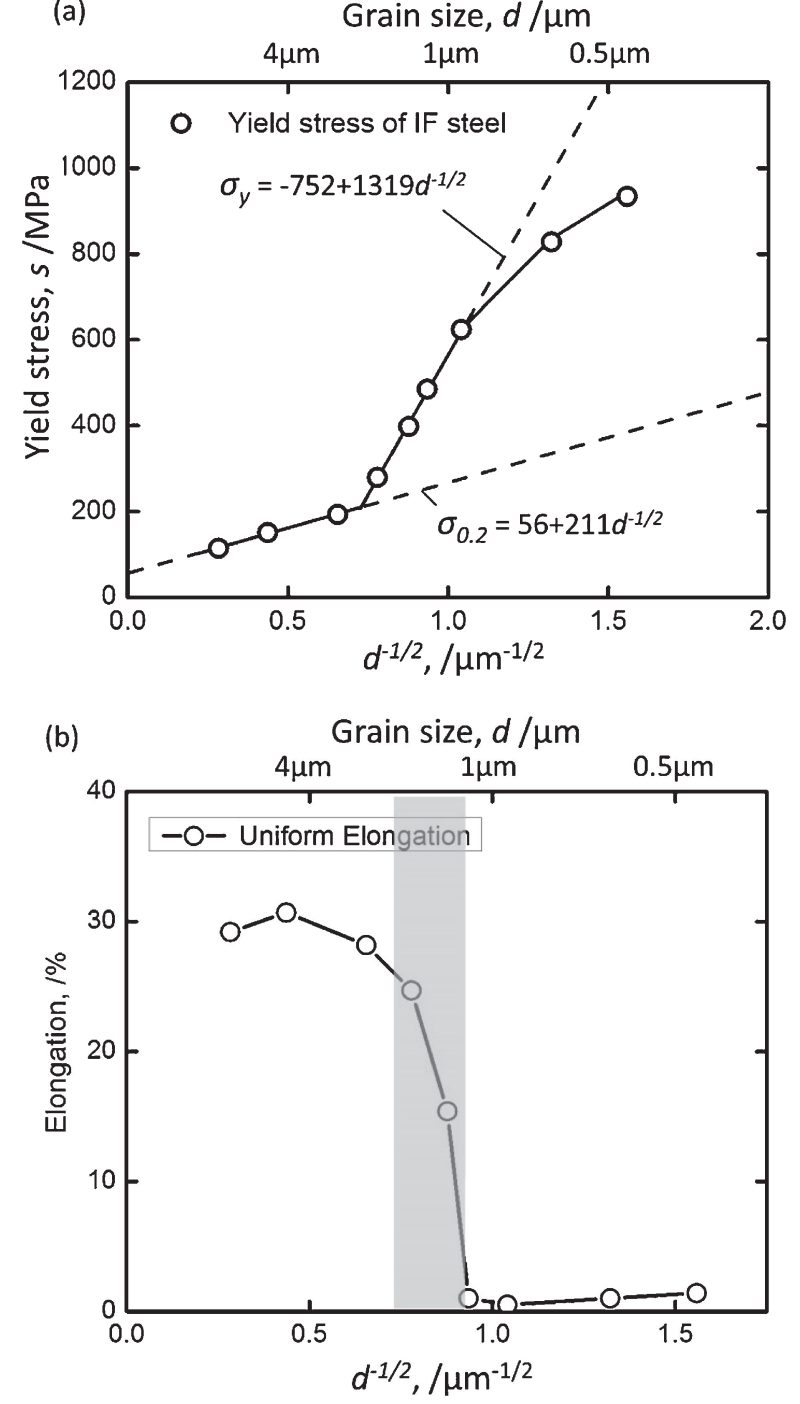

Fig. 3 Yield stress (a) and uniform elongation (b) of the IF steel plotted as a function of inverse square root of the mean grain size. The Hall-Petch relationships obtained from the specimens showed continuous or discontinuous yielding behaviors are also shown in different broken lines in (a).

point phenomenon in the fine grained IF steel in the present study, since the interstitial atoms are fixed by titanium through forming carbide and nitride. Besides the present study, the yield point phenomenon has also been found in other ultrafine grained materials including pure (99 and $99.99 \%$ purity) $\mathrm{Al}^{4,9)}$ pure $(99.97 \%) \mathrm{Cu},{ }^{10)}$ commercial purity $\mathrm{Ti}^{11}{ }^{11}$ various $\mathrm{Al}$ alloys ${ }^{12)}$ and austenitic steels, ${ }^{13,14)}$ all of which do not usually show the yield point phenomenon in coarse grain sizes. Therefore, it is considered that the yield point phenomenon is an unique mechanical property of the ultrafine grained materials. According to Johnston and Gilman's theory, ${ }^{15)}$ the yield point in pure metals is attributed to the lack of initial mobile dislocations. It is believed that Johnston and Gilman's theory can be applied to the yield point in ultrafine grained materials as well, ${ }^{12}$ although the mechanism of this interesting phenomenon has not been fully clarified yet.

As shown in Fig. 3(a), positive deviation from Hall-Petch relationship of the yield stress was found occurs when the

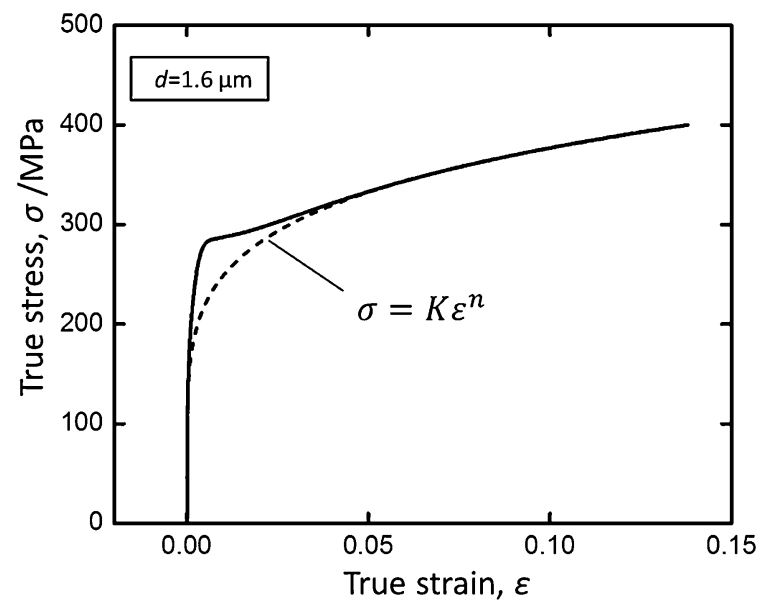

Fig. 4 True stress-strain curve and extrapolated stress-strain curve fitted by Hollomon equation for the IF steel specimen having the mean grain size of $1.6 \mu \mathrm{m}$.

grain size is smaller than $2 \mu \mathrm{m}$, which exactly coincides with the grain size below which the specimen starts to exhibit the yield point phenomenon. As the mean grain size further decreased, the yield point phenomenon became more prominent and the deviation from the Hall-Petch relationship for coarse-grained specimens also increased. These results suggest that the deviation of the yield stress from the HallPetch relation is caused by the yield point phenomenon. The yield point phenomenon, also referred to as the discontinuous yielding, is accompanied by the inhomogeneous deformation characterized by strain localization such as Lüders band. For the yielding occurring in such an inhomogeneous manner, a higher stress is required in order to nucleate and propagate such localized deformation bands. Since the plastic strain is localized during inhomogeneous yielding, the measured average strain throughout the gauge length cannot represent the true strain in the areas within which the plastic deformation actually occurs. Therefore, the actual flow behavior of the material during discontinuous yielding cannot be directly obtained from the macroscopic stress-strain curve. In order to estimate the true flow behavior of the material, the extrapolation process was carried out by using the Hollomon equation: ${ }^{16)}$

$$
\sigma=K \varepsilon^{n}
$$

where $\sigma$ is the true flow stress, $K$ is a strength index, $\varepsilon$ is the true strain and $n$ is a strain hardening exponent. The extrapolation process on one of the present specimens $(d=1.6 \mu \mathrm{m})$ is illustrated in Fig. 4. The work hardening region of the true stress-strain curve was fitted with the Hollomon equation and the fitted curve was extrapolated back to the elastic deformation region. From the fitted and extrapolated curves, the $\sigma_{0.2 \%}$ could be measured. The extrapolation process was performed on just two of the present specimens with the mean grain size of 1.3 and $1.6 \mu \mathrm{m}$, which showed clear yield point phenomenon and subsequent strain hardening. The extrapolation process could not be performed on the specimens whose mean grain size was smaller than $1.3 \mu \mathrm{m}$, since macroscopic necking and failure occurred immediately after the yield point and stress- 


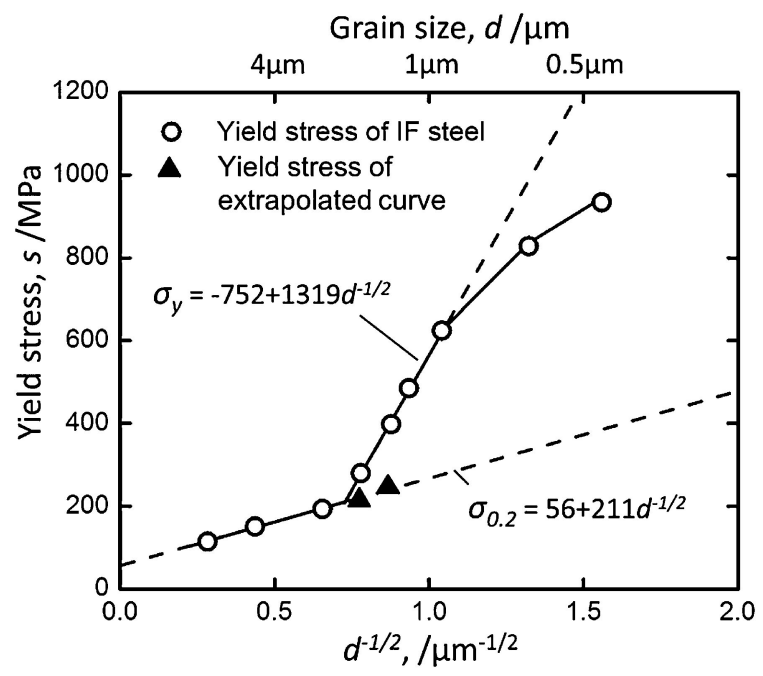

Fig. 5 Hall-Petch plots where the yield stresses $(0.2 \%$ proof stress $)$ obtained from the extrapolated stress-strain curve are added as closed triangles.

strain curves did not show strain hardening region. The $\sigma_{0.2 \%}$ measured from the extrapolated stress-strain curves was plotted as closed triangles in the Hall-Petch graph of Fig. 5. It has been found that the $\sigma_{0.2 \%}$ obtained from the extrapolated stress-strain curves seems to accord with the original Hall-Petch relation obtained from the coarse grain size specimens. This result clearly indicates that the positive deviation of the yield stress in the Hall-Petch graph is attributed to the yield point phenomenon.

Although it is shown that the positive deviation of the yield stress in the Hall-Petch relation in the fine grain size region is attributed to the yield point phenomenon, it is noteworthy that the deviated yield stress seems to follow a Hall-Petch type relation with a much higher slope and a negative interception as indicated in Fig. 5. The physical meaning of this linear relationship is still not clear, but it should be related to the discontinuous yielding behavior as well. The yield stresses of the as-ARB processed specimen and $500^{\circ} \mathrm{C}$ annealed specimen $(d=0.41$ and $0.57 \mu \mathrm{m})$ are found lower than those predicted by this linear relationship. This is possibly because these two specimens have such microstructures that are associated with large number of mobile dislocations, which may lower the flow stress at the yield point. Further investigation is necessary to clarify the physical meaning of the apparent Hall-Petch relation for the extra hardening plots.

As shown in Fig. 3(b), the uniform elongation of the material slightly increases first and then decreases as the mean grain size decreases from 12 to $2 \mu \mathrm{m}$. However, it suddenly drops to less than $2 \%$ as the mean grain size decreases from $2 \mu \mathrm{m}$ to about $1 \mu \mathrm{m}$, and then remains at a very low value (1-2\%) with further decreasing the mean grain size to submicron scale. Such a sudden drop in the uniform elongation is explained also by the Hall-Petch analysis of the yield stress and the flow stress, which is shown in Fig. 6. In Fig. 6, the yield stress, 0.05, 0.1, 0.15 and 0.2 flow stresses (true stress at each true strain) of the specimens having different mean grain sizes are plotted as a function of the minus square root of the mean grain size. It is

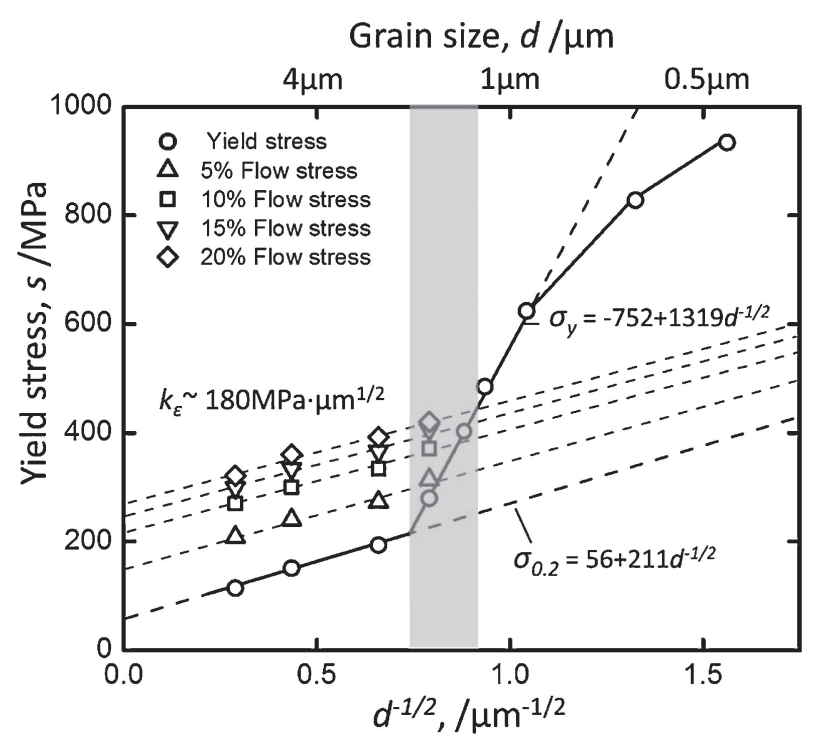

Fig. 6 Flow stresses at $0.05,0.1,0.15$ and 0.2 true strain plotted against the inverse square root of the mean grain size. The Hall-Petch slopes for the flow stresses at different strains show similar values of around $180 \mathrm{MPa} \cdot \mu \mathrm{m}^{1 / 2}$. The gray area indicates the grain size range where yield point phenomenon occurs and the material loses uniform elongation.

known that the Hall-Petch relation can be applied not only for the yield stress but also for the flow stress at a given strain: ${ }^{17}$

$$
\sigma_{\varepsilon}=\sigma_{0 \varepsilon}+k_{\varepsilon} d^{-1 / 2}
$$

where $\sigma_{\varepsilon}, \sigma_{0 \varepsilon}$ and $k_{\varepsilon}$ are the flow stress, friction stress and Hall-Petch slope at a given strain $(\varepsilon)$, respectively. It can be seen in Fig. 6 that, in the coarse grain size region, the flow stresses at different strains also follow the Hall-Petch relation (dashed lines in Fig. 6) having nearly an identical slope (about $180 \mathrm{MPa} \cdot \mu \mathrm{m}^{1 / 2}$ ) and increasing $\sigma_{0 \varepsilon}$ with increasing the strain. When the mean grain size decreases below $2 \mu \mathrm{m}$, the yield stress starts to deviate from the HallPetch relation for coarse grains, and even exceeds the flow stresses at given strains. If the yield stress exceeds the flow stress, it indicates that strain softening (or plastic instability) occurs at the onset of yielding. The plastic instability proceeded in the Lüders deformation manner when the mean grain size was between 1.3 and $1.2 \mu \mathrm{m}$. In this grain size range, strain hardening still occurred after Lüders deformation. With decreasing the mean grain size furthermore, the deviation of the yield stress from the original HallPetch curve increased rapidly within a very narrow grain size range (indicated by the gray area in Fig. 6) and it even exceeded the flow stress at a 0.2 strain. It should be noted here that the narrow gray region shown in Fig. 6 coincides with the narrow grey region where the uniform elongation suddenly drops to a lower level in Fig. 3(b). That is, the loss of uniform elongation and plastic instability occur in the same narrow grain size range. This means that the abrupt drop of uniform elongation in fine grained materials can be predicted also by the Hall-Petch graph shown in Fig. 6 . When the mean grain size becomes smaller than $1.3 \mu \mathrm{m}$, Lüders strain becomes significantly high, so that necking immediately occurs within the first Lüders band. Then the uniform elongation sharply drops to below $2 \%$. 


\section{Conclusion}

In the present study, we fabricated the IF steel specimens with different grain sizes ranging from 0.4 to $12 \mu \mathrm{m}$ by ARB process and subsequent annealing. Tensile tests at room temperature revealed that with decreasing the mean grain size down to an ultra-fine range, the yielding behaviors gradually changed from the continuous yielding to the discontinuous yielding accompanying a yield drop. As the mean grain size became smaller than $2 \mu \mathrm{m}$, the yield stress deviated from the original Hall-Petch relation extrapolated from the coarse grain size range. The deviation of the yield stress was attributed to the discontinuous yielding phenomenon, which was confirmed by the Hall-Petch analysis using extrapolated stress-strain curve. The Hall-Petch analysis also implied that the abrupt loss in the uniform elongation in the ultrafine grain size range corresponded to the appearance of discontinuous yielding behavior. As the discontinuous yielding has been found in many UFG materials, we believe that it is an unique mechanical behavior of UFG materials. And the present study has shown that it has a significant importance on the mechanical performance of ultrafine grain materials.

\section{Acknowledgement}

This study was financially supported by the Grant-in-Aid for Scientific Research on Innovative Area, "Bulk Nanostructured Metals" (area No. 2201), the Grant-in-Aid for Scientific Research (A) (No. 24246114), and the Elements
Strategy Initiative for Structural Materials (ESISM), all through the Ministry of Education, Culture, Sports, Science and Technology (MEXT), Japan (contract No. 22102002), and the supports are gratefully appreciated.

\section{REFERENCES}

1) E. O. Hall: Proc. Phys. Soc. B 64 (1951) 742-747.

2) N. J. Petch: J. Iron Steel Inst. 174 (1953) 25-28.

3) A. H. Cottrell and B. A. Bilby: Proc. Phys. Soc. A 62 (1949) 49-62.

4) H. Takechi: ISIJ Int. 34 (1994) 1.

5) N. Tsuji, Y. Ito, Y. Saito and Y. Minamino: Scr. Mater. 47 (2002) 893899.

6) N. Tsuji, R. Ueji and Y. Minamino: Scr. Mater. 47 (2002) 69-76.

7) N. Kamikawa, N. Tsuji and Y. Minamino: Sci. Tech. Adv. Mater. 5 (2004) 163-172.

8) S. Takaki, K. Kawasaki and Y. Kimura: J. Mater. Process. Tech. 117 (2001) 359-363.

9) N. Kamikawa, X. Huang, N. Tsuji and N. Hansen: Acta Mater. 57 (2009) 4198-4208.

10) H. An, S. Wu, Z. Zhang, R. B. Figueiredo, N. Gao and T. G. Langdon: Scr. Mater. 66 (2012) 227-230.

11) Z. Li, L. Fu, B. Fu and A. Shan: Mater. Lett. 96 (2013) 1-4.

12) C. Y. Yu, P. W. Kao and C. P. Chang: Acta Mater. 53 (2005) 40194028.

13) T. Wang, Z. Li, B. Zhang, J. Deng and F. Zhang: Mater. Sci. Eng. A 527 (2010) 2798-2801.

14) R. Saha, R. Ueji and N. Tsuji: Scr. Mater. 68 (2013) 813-816.

15) W. G. Johnston and J. J. Gilman: J. Appl. Phys. 30 (1959) 129-144.

16) W. L. Phillips and R. W. Armstrong: Metall. Mater. Trans. B 3 (1972) 2571-2577.

17) R. Armstrong, I. Codd, R. M. Douthwaite and N. J. Petch: Philos. Mag. 7 (1962) 45-58. 